\title{
Abstract Clustering for Program Comprehension
}

\author{
Christophe Tabacznyj ${ }^{1}$ \\ Leti (CEA - Technologies Avancées), DEIN/SLA (Bât. 528), F 91191 Gif-sur-Yvette \\ Loria (Université Henri Poincaré - Nancy 1), BP 239, F 54506 Vandoeuvre-lès-Nancy \\ Christophe.Tabacznyj@loria.fr
}

\begin{abstract}
A new formal framework for program comprehension, Abstract Clustering, is presented. To manage the process of semantic-based modularisation of a software system from its source code, Abstract Interpretation is used to find a partition rather than measures. The result is a formal, semantic-based framework that offers a new approach to program comprehension.
\end{abstract}

\section{Introduction}

The goal of program comprehension is to extract sufficient knowledge about a software system to identify and locate relevant portions of code according to evolution or verification requirements. It is a guide during reverse-engineering activities which are usually resulting of a lack or a lost of design documentation of a software system (information may be out of date or no longer available, a long period of maintenance may have broken the consistency of the original software design for example). Our concern for program comprehension is motivated by verification purposes [1]. Hence, more than extraction of knowledge, we need to work using formal methods to be able to re-use the extracted information during verification process. The use of support mechanisms to manage the complexity of programs by helping the software engineer to extract high-level information from low-level code is a central need. The essence of program comprehension is identifying semantic-components and understanding their relationships mainly by semantic-pattern matching at various abstraction levels. It involves to identify, to manipulate, and to explore semantic-components for a particular subject system representation via pattern recognition and the aggregation of these semantic-components to form more abstract system representations [13].

Clustering (or data gathering) starts the reverse-engineering process, and then program comprehension relies merely upon exploration of the abstractionspace. Knowledge management is needed to structure data into a model of the application domain. Our contribution relies on the systematic and formal use of clusters from a semantic point of view; they reduce complexity and simplify the structure by clustering source-domain elements together to form more abstract, aggregate objects. Clusters represent sets of source-elements as unique semantic entities.

Complexity of large software systems results of the interleaving of their components by several kinds of relationships having their own meaning. During program comprehension, software engineers have to navigate through this semantic 
knot, to locate relevant code fragments that implement concepts (particular semantics) in the subject domain. This semantic-pattern recognition is performed mechanically by our Abstract Clustering Framework. Semantic-components can be selected according to various criteria based on their semantics. If patterns can be represented in terms related to semantics, then the software engineer can verify more easily the program. Our methodology can be integrated in a tool or leveraged as an external tool to provide advanced searching techniques and having results of their searches made available to the user and the environment.

Abstract Clustering Framework methodology may be seen as a formal refinement of "a reverse engineering environment framework" presented by Scott Tilley in [13]. It is also a generalization of automatic modularisation of code as exposed in [11] by S. Mancoridis et al. Moreover, the formal foundation of this work relies on Abstract Interpretation, a theoretical framework for program analysis introduced by Patrick and Radhia Cousot in their seminal papers [6, 7].

In section 2, principles of Abstract Clustering are introduced from subject domain to sound modularisation via abstraction and its domain. Section 3 develops Abstract Clustering analysis defining its scheme, its basic algorithm and the way to treat hierarchical clustering. Abstract Clustering Framework appears in section 4 when studying organization of Abstract Clustering. A case study, Type clustering, is examined in section 5 and then, after a comparison with related work, we conclude on reverse-engineering qualities inherent to our formal framework.

\section{Principles of Abstract Clustering}

Software source-code is a set of components together with relationships between them; the most complete (and over-detailed) description available of this system is given by its operational semantics, which describes how the program is executed. Representative types of components/relationships presentations include classes/inheritance, procedures/call-graph, variables/dependencegraph, data and control flow, and so on. Our goal is to define an automatic modularisation process according to one of these classes of knowledge presentation, which preserves soundness of the information extracted. By this, we mean that the system partition into clusters (subsystems) can be taken as a safe basis for reasoning, and what we can conclude about such a clustering remains (formally) true for the whole system.

To achieve this semantic modularisation, we treat clustering as an abstract interpretation analysis [6,7], where the goal is to extract sound semantic information. Clusters represent higher-level component abstractions of the system. Each subsystem contains a set of elements of the subject domain.

Abstract Clustering is a central support mechanism to aid program comprehension. It is a process of semantic examination without alteration of the subject system. It helps program understanding by formally supporting its principles: identifying semantic-components, discovering relationships, and generating abstractions as clusters. Components manipulated are classified in: 
- Set of elements: concrete data used as the basis for analysis.

- Partial-order: the knowledge, which captures the semantic relationships between data.

- Partially ordered clusters: contextually extracted knowledge from an abstract semantic point of view.

Elements of the subject domain are the foundation for the higher-level knowledge semantic-components during program comprehension.

Abstract Clustering allows different levels of abstraction parameterised by semantics [4,3]. Source code, structural and functional relationships, and applicationdomain oriented concepts can be investigated by assigning abstract values to semantic patterns. Because different levels of analysis are useful for different program understanding purposes, different abstract clustering can be combined and composed allowing the end-user to consider source code in an increasingly abstract forms - and soundness is preserved over all this hierarchy of abstractions. More abstract forms entail and locate higher semantic information, bridging the gap between the code side and the meaning side, between what it is and what was intended to do.

\subsection{Subject Domain}

The subject domain - or concrete domain - is the domain to analyse while the abstract domain represents the abstraction, which gives the semantics. We define an abstract clustering as an equivalence partition of the subject domain over the semantic values of the abstract domain. Two elements of the subject domain are in the same cluster if they have the same abstraction. The relationship between clusters is the abstract relationship between abstractions.

At this point, we need to define formally the (generic) structure and operations of a domain, and the relationship between domain elements. From a set theory point of view, we would like to reason on a structure like the powerset $\wp(S)$ of a set $S$, with intersection $\cap$ and union $\cup$ operations between subsets of $S$. Therefore, what relationship between two subsets allows us to compare them? Inclusion $\subseteq$; all subsets are partially ordered by it. Any subset $X$ is included in $S$ (supremum, $S \in \wp(S)$ ) and contains the empty set $\emptyset$ (infimum, $\emptyset \in \wp(S)$ ) such as $\forall X \in \wp(S), \emptyset \subseteq X \subseteq S$. Moreover, any set $\mathcal{S}$ of subsets of $S$ has an intersection $\cap \mathcal{S}=\cap\left\{\left\{S_{i}\right\}_{i \in I}\right\}=\cap_{i \in I} S_{i}$ and a union $\cup \mathcal{S}$. Then we have defined the structure $\wp(S)(\subseteq, \cap, \cup, \emptyset, S)$ which is, from an algebraic point of view, a complete lattice $[16]$ :

Definition 1. A complete lattice $L(\subseteq, \cap, \cup, \perp, \top)$ is a set $L$ partially ordered by a partial order $\subseteq$ with meet $\cap$ and join $\cup$ operations, infimum $\perp$ and supremum $\top$ elements, such as every subset $X$ has a meet $\cap X$ and a join $\cup X$.

So, we regard the subject domain as being a complete lattice, which means we are able to manipulate clusters (subsets of elements) simply using set-like operations. An example of complete lattice is the powerset of a set. 


\subsection{Abstraction and its Domain}

Now, we define the process of clustering, following the main features of semantic analysis of programs [6,7],[8]. The operational semantics of a program $P$ can be specified by a relation on a set of states $S$. One can characterize the set of reachable states of $P$, as the solution of the equation $\mathcal{F}(X)=X$ where $\mathcal{F}$ is an isotonic ${ }^{1}$ operator on the powerset $\wp(S)$ (partially ordered by set inclusion) which computes the behaviour of $P$ as intersections and/or unions of subsets of $S$. Then, the set of reachable states is equal to the least fix-point $\mu \mathcal{F}$ of $\mathcal{F}$ in the complete lattice $\wp(S)(\subseteq)-\mu \mathcal{F}$ is called collecting semantics. This characterization of $\mu \mathcal{F}$ makes possible to compute it by iteration, but this may be infinite (it is an undecidable problem). The principle of semantic analysis of programs is to make this computation possible by replacing the subject domain - the complete lattice $\wp(S)(\subseteq)$ - by an abstract one, an $\mathcal{F}$ by an abstract operator $F$ on the abstract domain, in such a way that the corresponding iteration terminates, yielding an abstract but sound result. This can be done using a pair of adjoined functions $\langle\alpha, \gamma\rangle$ where $\alpha$ maps subject-domain elements to abstract ones and $\gamma$ does the reverse mapping. More formally, we need a Galois connection [6,7]:

Definition 2 (Galois Connection). Let $\mathcal{M}(\subseteq)$ and $L(\sqsubseteq)$ be two complete lattices. A Galois connection is a pair of adjoined functions $\langle\alpha, \gamma\rangle: \mathcal{M}(\subseteq) \underset{\alpha}{\stackrel{\gamma}{\rightleftarrows}}$ $L($ ㄷ) such that:

$$
\forall x \in \mathcal{M}, \forall y \in L, x \subseteq \gamma(y) \Longleftrightarrow \alpha(x) \sqsubseteq y
$$

$\alpha$ is called abstraction function and $\gamma$ concretization function.

Then we can formalize results about collecting semantics:

Proposition 1 (Least fixpoint of $\mathcal{F}$ ). Let $\mathcal{F}(\subseteq)$ be an isotonic operator on $\mathcal{M}(\subseteq)$ then $F=\alpha o \mathcal{F}$ o $\gamma$ is isotone on $L(\sqsubseteq)$ and $\mu \mathcal{F} \subseteq \gamma(\mu F) \Longleftrightarrow \alpha(\mu \mathcal{F}) \sqsubseteq$ $\mu F$.

So, when the subject-domain $\mathcal{M}(\subseteq)$ is the lattice of sets of states designed by $\wp(S)(\subseteq)$, the set $\gamma(\mu F)$ can stand for the set of reachable states; moreover, if the complete lattice of semantic properties of $P$ designed by $L(\sqsubseteq)$ is finite for example, then $\mu F$ can be calculated as the result of an iterative computation ${ }^{2}$. To our concerns, the design of a semantic analysis relies on the choice of a suitable abstract domain $L(\sqsubseteq)$.

\subsection{Sound Modularisation}

During program comprehension, software engineers look for code that fits certain patterns depending on the meaning - the semantics - they are considering. To discover and locate instances of abstract representations in the subject

\footnotetext{
${ }^{1}$ isotone means $X \sqsubseteq Y \Rightarrow \mathcal{F}(X) \sqsubseteq \mathcal{F}(Y)$ on the same domain.

${ }^{2}$ cf Tarski's least fixpoint theorem $\left(\mu f=\bigcup_{n} f^{n}(\perp)\right)$.
} 
domain, a mechanism of semantic-pattern recognition is required. Semanticpatterns are abstract representations of source code fragments. This process involves semantic-pattern matching to focus on recognition of similar code fragments. These characteristics are used to classify two code fragments according the abstract ordering over their abstractions [13].

Now, let $S$ be a set and $\wp(S)(\subseteq)$ our subject domain obtained as the powerset of $S$ ordered by set inclusion. Then, one can define a semantic equivalence relation $\stackrel{\alpha}{=}$ between the elements of $S$ such that:

$$
\forall x, y \in S, \quad x \stackrel{\alpha}{=} y \Longleftrightarrow \alpha(x)=\alpha(y)
$$

Let $S / \underline{\underline{\alpha}}$ be the quotient set of $S$ by $\stackrel{\alpha}{=}$. Then $S / \underline{\underline{\alpha}}$ is a partition ${ }^{3}$ of $S$ in clusters of elements which have the same abstraction. So, we have the relations ${ }^{4}$ of figure 1 :

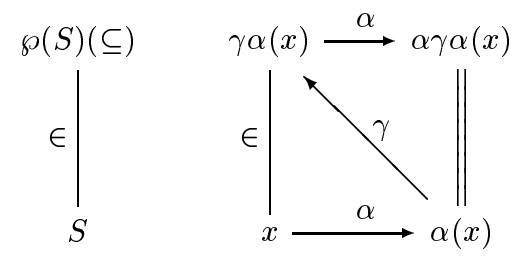

Fig. 1. Abstraction Relations.

This diagram means that:

$-x$ is an element of the subject domain,

- its abstract corresponding element is $\alpha(x)$ in the abstract domain,

$-\gamma \alpha(x)$ represents the set of elements of the subject domain which have the same abstraction $\alpha(x)$ than the element $x$; then $x \in \gamma \alpha(x)$,

$-\alpha \gamma \alpha(x)$ is the abstraction of $\gamma \alpha(x)$ which is in a sense the set of corresponding abstractions of $\gamma \alpha(x)$ and this is equal to $\alpha(x)$ by construction.

In the next section, we will call $\gamma \alpha(x) \in \wp(S)(\underline{\subseteq})$ a cluster and $\alpha \gamma \alpha(x) \in \alpha(S / \underline{\underline{\alpha}})$ a (cluster) class.

\section{Abstract Clustering Analysis}

To identify semantic-patterns and relationships of a subject domain and use them to construct and explore higher-level abstractions, subject-domain elements have

$\overline{3}^{3}$ a family $\left\{S_{i}\right\}_{i \in I}$ of subsets of $S$ is a partition of $S$ iff $\bigcup_{i \in I} S_{i}=S$ and $\forall i, j \in I, i \neq$ $j, S_{i} \cap S_{j}=\emptyset$

4 the notation $x \in \gamma \alpha(x)$ is an abuse of language therefore useful for understanding. We should read $\{x\} \subseteq \gamma \alpha(\{x\})$ 
to be gathered. A critical point is to gather together elements without misinterpretation [13]. So, such a process must be formal because it is the basis upon which most abstract representations of the system are built. The amount of data gathered for understanding large systems can be large, and easily exceed our capacity to treat it. Abstract Clustering makes a decision between what to keep and what to ignore according to semantics, so it plays the role of a formal data-filtering technique in aiding program comprehension. It is used to extract selected semantic-patterns and relationships from a large data source. To build our initial subject domain (the source), we need to parse the source code of the system. Such a standard syntactic analysis can be performed by either writing a (LALR) parser (with Lex \& Yacc generators for example) or reusing existing tools; we have chosen the later solution using lcc [10], a retargetable compiler for C ANSI code, which provides us the abstract syntax tree and its compiling environment (symbol table, ...). Then, such an abstract syntax tree can be used as a subject domain, whose syntactic nodes represent subject domain elements and tree structure defines the partial order ${ }^{5}$.

\subsection{Clustering Analysis}

Through abstract interpretation of the subject domain, Abstract Clustering allows one to capture, to order modulo a meaning and so to present a more understandable organization of data. Each abstract clustering creates a domain model, which is a representation that captures the structure and composition of semantic-patterns for an interpretation.

As announced at the end of the previous section, we define a partition of $S$ as a set of clusters which are subsets of elements of $S$ having the same abstraction $\alpha(x)$ for a given interpretation $A(\alpha, \gamma)$. A cluster is an equivalence class for the equivalence relation $\stackrel{\alpha}{=}(\forall x, y \in S,[x]=[y] \Longleftrightarrow x \stackrel{\alpha}{=} y \Longleftrightarrow x, y \in \gamma \alpha(x) \equiv$ $\gamma \alpha(y))$. So, the definition is:

Definition 3 (Cluster). The cluster for an abstract interpretation $A(\alpha, \gamma)$ of an element $x$ of the subject domain is the equivalence class of $x$, denoted by Cluster $(A, x)$.

Then, a class is the abstract value associated to a cluster $C$, such that $\forall x, y \in$ $C, \alpha(x)=\alpha(y)=$ class $(C)$. Moreover, a class, like a cluster, relies on an interpretation:

Definition 4 (Class). The class for an abstract interpretation $A(\alpha, \gamma)$ of an element $x$ of the subject domain is the abstract domain element $\alpha(x)$, denoted by $\operatorname{Class}(A, x)$.

Thus, by transposing the diagram of the figure 1, we have the relations of figure 2 .

This diagram shows the foundational properties of Abstract Clustering. An element $x$ of the subject domain is a $\operatorname{Class}(A, x)$ element in the abstract domain

\footnotetext{
${ }^{5}$ nested blocks of declarations and instructions.
} 


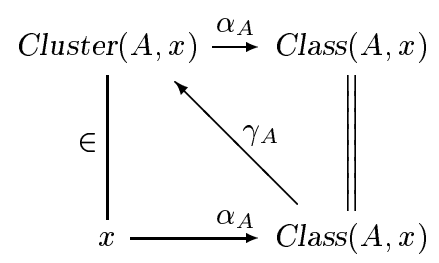

Fig. 2. Abstract Clustering.

and is part of the Cluster $(A, x)$ which contains all elements of the same class. Moreover, $\operatorname{Cluster}(A, x)$ is an instance of $\operatorname{Class}(A, x)$ in the subject domain. Finally, $\operatorname{Class}(A, x)$ designs both the abstract element corresponding to an element and the one corresponding to a class. The intuition is simple. For example, if we consider simply typed variables of a $\mathrm{C}$ like program, let $x$ be a variable of type integer int, then the class of $x$ is int and the set of declared variables of type int is Cluster $_{\text {int }}\left(\mathcal{T}_{\text {ype }}, x\right)$. Such a cluster is an instance of the class of integer values so the equality with int. The small diagram follows:

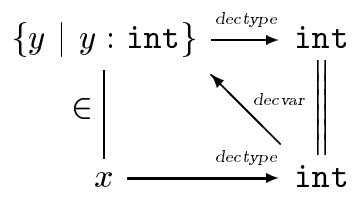

The associated interpretation is $\mathcal{T}_{\text {ype }}$ (dectype, decvar) where dectype returns the declared type of a given variable and decvar returns declared variables of a given type. In the next section, we present how to compute a partition.

\subsection{A Clustering Algorithm Scheme}

Now, we present the algorithm for determining the abstract clustering of a finite subject domain $S$ for an interpretation $A(\alpha, \gamma)$. This algorithm is straightforward, starting with the most precise but less information carrying partition $I$, which is one cluster for each element. The resulting set is a multiple set from the abstract side since different clusters may have the same abstraction. So we sum (U) together clusters whose elements are in connection with the same abstract value. The result of this process is the abstract clustering $P$.

Then, to evaluate the properties for an interpretation $A(\alpha, \gamma)$ of an isotonic operator $\mathcal{F}$ defined on a subject domain $\mathcal{M}(\subseteq)$, one can proceed by replacing elements by clusters and operators on elements by operators on clusters - the intuition is that a property $P_{A}$ for an interpretation $A$ is preserved by aggregation of elements of the class: $P_{A}(x, \mathcal{F})=P_{A}(\operatorname{Cluster}(A, x), F)$.

Therefore in practice, if $\mathcal{F}$ is defined as a functional $-\mathcal{F}=\mu(X=\mathcal{G}(X))$ then, when the abstract domain is not complete or finite for example, the least 
1. Let $S=\left\{e_{1}, e_{2}, \ldots, e_{n}\right\}$ be the subject domain, and $\subseteq$ be the graph representing relationships between elements in $S$.

2. Compute $I_{A}=\{\langle x, \alpha(x)\rangle \mid x \in S\}$, then

3. $P_{A}=\left\{\operatorname{Cluster}(A, x)=\bigcup_{\langle y, \alpha(x)\rangle \in I_{A}}\{y\} \mid x \in S\right\}$

Fig. 3. Clustering Algorithm Scheme.

fix-point may not be reached or only after infinitely many steps. To accelerate or make effective the convergence of the fix-point, $[6,7]$ introduced widening and narrowing operators. Widening is used during iteration to reach a post fixpoint $p F$ behind the least fix-point $\mu F \sqsubseteq p F$ and then narrowing is used to find another post fix-point $p^{\prime} F$ that is a better approximation by reducing the distance with the least fix-point in the lattice of fix-points $\mu F \sqsubseteq p^{\prime} F \sqsubseteq p F$. An accurate discussion about narrowing and widening operators can be found in [9].

\subsection{Hierarchical Clustering}

The algorithm presented in the previous section constructs the partition associated to an interpretation of a subject domain. Such a clustering recovers knowledge by gathering elements part of the same semantic information. However, the number of resulting clusters may be large and before thinking to a more filtering clustering or to design a second stage clustering (taking the abstract domain as subject one for the interpretation $B\left(\alpha^{\prime}, \gamma^{\prime}\right)$ - the composed interpretation is then $\left.A B\left(\alpha^{\prime} o \alpha, \gamma o \gamma^{\prime}\right)\right)$, one can apply recursively the current analysis, making clusters of clusters.

Definition 5 (Lower Moore Family). Let $L(\sqsubseteq)$ be a complete lattice and $E$ be a subset of $L$. Then $E$ is a lower Moore family when $E$ is $\wedge$-closed: $\forall S \subset$ $E, \wedge S \in E$.

Formally, if $C$ is a cluster, $\sqcap C=\{\gamma(X) \mid X \sqsubseteq \alpha(C)\}$ denotes its hierarchical completion (it is a lower Moore family of clusters ordered by abstract partial order). The idea is to collect downward the clusters over the abstract order: for example, if real is a the set of real valued variables of a program and int its set of integer valued ones, then as void $\sqsubseteq i n t \sqsubseteq$ real in the abstract domain, we have $\sqcap$ real $=$ real $\cup$ int $\cup$ void. Hierarchical Clustering is directly supported by Abstract Clustering. It raises a partition of the subject domain. Each cluster is in connection with an abstract element of the abstract domain. Then, the fact that the abstract domain is partially ordered is used; the abstract (partial) order $\sqsubseteq$ defines a hierarchy over the abstract element and then over the clusters. Hierarchical Clustering is embedded in Abstract Clustering through the partial order of the abstract domain. 


\subsection{On cofibered domains}

In [14], Arnaud Venet defines an abstract interpretation framework based on cofibered domain to perform analysis such that aliasing. We show here that our Abstract Clustering can be considered in terms of such domains. More formally, let $C a t$ be the category of small categories with functors, Proset be the category of preordered sets with monotone maps, and Poset be the category of partially ordered sets with monotone maps. Let $\Delta: C \rightarrow$ Poset be a functor from a small category $C$ into Poset, such that for each object $A$ of $C$, the poset $\left(\Delta A, \subseteq_{A}\right)$ is called the fibre of $\Delta$ over $A$. The Grothendieck construction associates to $\Delta$ the category $G \Delta$ defined as follows:

- $\operatorname{Obj}(G \Delta)=\{(A, x) \mid x \in \Delta A\}$

$-\operatorname{Mor}(G \Delta)=\left\{f:(A, x) \rightarrow(B, y) \mid f \in \operatorname{Hom}_{C}(A, B) \wedge \Delta f(x) \leq_{B} y\right\}$

Let $U: C a t \rightarrow$ Proset be the forgetful functor that sends every category to its underlying preordered set obtained by collapsing each hom-set into one arrow.

Definition 6. A cofibered domain is a preordered set $P=(E, \preceq)$ such that there exists a functor $\Delta C \rightarrow$ Poset verifying $P=U G \Delta$. The functor $\Delta$ is called the display of the domain and $C$ its base.

So, if we interpret $(A, x)$ as $(\alpha(x), x)$, then $\Delta A=C l u s t e r(x)$. A clustering domain is then, categorically speaking, a cofibered domain. Our approach gives a generic construction of cofibered domains from an abstract interpretation framework. Arnaud Venet uses cofibered domain as a starting point to formalize more pathological analyses (alias, communications).

\section{Abstract Clustering Framework}

When semantic-pattern recognition has located source code fragments of interest for the software engineer, program semantic-patterns have not only to be returned in terms of clusters of the subject domain. Indeed, meaning-level concepts are also represented by abstract values. The level of analysis is directly related to the abstraction of semantic-components being manipulated; there are many abstractions and so many analyses possible. Various abstractions can be used to guide the creation of new semantic-components in the information space.

Therefore, to do so we need to have a deeper understanding of relationships between Abstract Clustering and a framework to organize them, as various abstractions and level of abstractions. We will not study here the organization of multiple abstractions in the information space. Therefore, it is interesting to specify data organization of a particular clustering analysis.

\subsection{Abstract Clustering Framework}

From definition of Abstract Clustering (section 3.1), we can induce the principles of an Abstract Clustering Framework. We give some insights on the Abstract 


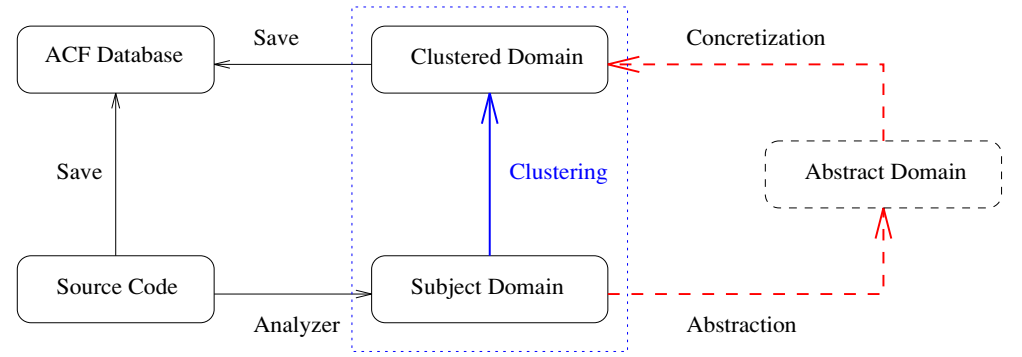

Fig. 4. Clustering Framework Environment.

Clustering Framework implementation, the way to design it effectively. Figure 4 shows entities which compose the Abstract Clustering Framework.

The basement of the analysis is formed of the source code, which is parsed by an analyser to produce the root subject domain. By clustering, a clustered domain is generated; both source code and clustered domain are saved in the Abstract Clustering Framework database. At the next step, source code will be replaced by the subject domain, the analyser by identity function, so both subject domain and clustered domains will be saved in the Abstract Clustering Framework database.

Principles diagram, environment and database organization define an $A b$ stract Clustering Framework. Now, we precise how a particular Abstract Clustering is represented as a database.

\subsection{Abstract Clustering Database}

For program comprehension, data must be in a form that facilitates their management, allows analysis of semantic-patterns and relationships, and reflects system characteristics seen by the user [13]. This need to organize data in some well-defined and formal manner led to the development of a data model. A large database needs organizational principles to be understandable, just like a large software system. Abstraction is the keystone concept used for organizing information; it is the selective emphasis on pertinent details to the problem whereas others are forgotten. Such a mechanism serves as an organizational axe for structuring the database focusing on high-level aspects of an entity while concealing details. Common abstraction mechanisms can be easily identified in the Abstract Clustering model (see figure 5):

Classification A class is defined as a cluster which is a set of instances. Elements of each cluster share a common abstraction, resulting in an abstract element which captures the semantic similarity among its constituents. An instance-of relationship is established between a class in the abstract domain and every instance in the subject domain.

Aggregation A relationship between elements is considered as a cluster. When considering the cluster, specific details of the subject elements are sup- 
pressed. A part-of relationship is established between subject elements and the cluster itself.

Generalization Similar elements are related to a class. The constituent elements are considered as specializations of the class. An $i s-a$ relationship is established between specialized elements and the class.

Identification Both definitions of a class, as an abstract element or as an abstract cluster, capture the same semantic-information. An $i d$ relationship is established between the class of an element and the class of its associated cluster.

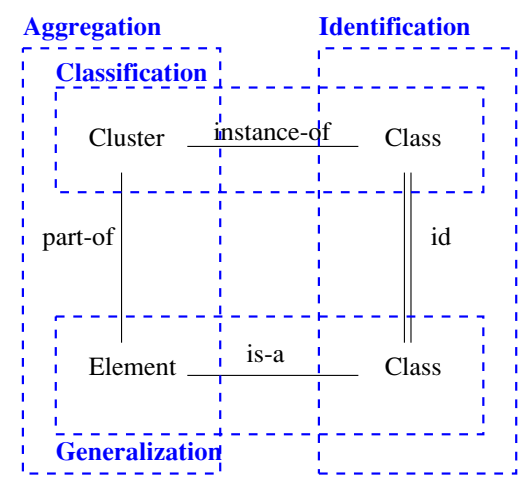

Fig. 5. Clustering Data Model.

Such a data model (figure 5) emphasizes on semantic organization rather than on data organization. Abstract Clustering provides a formal model that fits requirements for organizing three different categories of semantic-components (data as subject domain elements, knowledge as partial order, and information as partially ordered clusters) in a single representation technique.

\section{Example: Type Clustering}

In this section we first study the case of Type clustering introduced in section 3.1. Let $\mathcal{X}$ be a set of variables and $\wp \mathcal{X}(\subseteq)$ the powerset of variables ordered by set inclusion. Let $\mathcal{T}_{\text {ype }}(\sqsubseteq)$ be a complete lattice of pre-defined (or declared) types, and let $\wp \mathcal{X}(\subseteq) \underset{\text { dectype }}{\stackrel{\text { decvar }}{\leftrightarrows}} \mathcal{T}_{\text {ype }}(\sqsubseteq)$ be a Galois connection as described in figure 6 . The associated interpretation is $\mathcal{T}_{\text {ype }}$ (dectype, decvar) where dectype returns the declared type of a given variable and decvar returns the declared variables of a given type. If we consider weakly typed variables of a $\mathrm{C}$ like program, let $x$ be a variable of type $\tau$, then the class of $x$ is $\tau$ and the set of declared variables of type $\tau$ is $\operatorname{Cluster}_{\tau}\left(\mathcal{T}_{\text {ype }}, x\right)$. Such a cluster is an instance of the class of $\tau$ values so the equality with $\tau$. The small diagrams of figure 6 follows. 


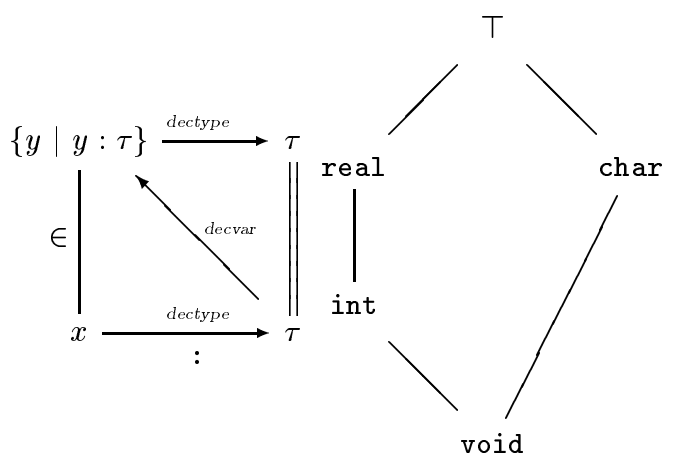

Fig. 6. $\mathcal{T}_{\text {ype }}(\sqsubseteq$, void $, \top, \sqcup, \sqcap)$ Clustering and Lattice.

The first diagram reminds the relationships for this particular clustering while the second one represents the complete lattice of types $\mathcal{T}_{\text {ype }}(\sqsubseteq)$ restricted to types int, real and char for our example. Now, in order to simplify the notations, we will use a path notation to denote scope information about variables in a software system (e.g. file.c/proc/y means variable y of procedure proc of file file.c of the software system). Each contextual declaration of a variable (scope-pathed variable) is clustered according its type.

More formally, let figure 7 present the syntax and the operational semantics of a simple imperative language where: $x \in \mathcal{X}:$ program variable, e,exp $\in \mathcal{E}$ : program expressions, type $\in \mathcal{T}_{\text {ype }}$ : variable type, $b \in \mathcal{B}:$ Boolean expressions. For simplicity, Boolean expressions are implicitly defined. Moreover, type $x$ is a declaration of variable with type, (type) $x$ is a type conversion, if, ; , while and return are usual control flow commands, and exp is either a variable or a sum of expressions (note that + is overloaded according types: numerical addition for real and int, and concatenation for char). The semantic domain $\mathcal{S}$ is defined by (see figure 6):

$$
\mathcal{S}=\left[\text { int }_{\text {void }} \oplus \text { real }_{\text {void }} \oplus \text { char }_{\text {void }} \mid \text { int } \sqsubseteq \text { real }\right]
$$

where $D_{\text {void }}$ is the lift domain on types and $\oplus$ is the coalesced sum of domains. The semantics $S \llbracket \bullet \rrbracket \in \mathcal{E} \mapsto \mathcal{S}$, is defined by figure 7 (Convention: $E[x]=R \Longleftrightarrow$ $E=(E-\langle x, *\rangle) \cup\langle x, R\rangle)$.

then, its collecting semantics $C \llbracket \bullet \rrbracket \in \mathcal{E} \mapsto \wp(\mathcal{S})$ is the strongest program property $C \llbracket e \rrbracket=\{S \llbracket e \rrbracket\}$ on the lattice $\langle\wp(\mathcal{S}), \subseteq, \emptyset, \mathcal{S}, \cup, \cap, \neg\rangle$ with logical implication as subset inclusion $\subseteq$. Figure 8 defines its abstract semantics.

The abstraction function is just a forget functor that "forgets" values associated to variables and does not consider conditional statements of the control flow (when an if statement is encountered, the union of control flow branches is taken as the resulting abstraction and while loops are forgotten-the type of the inner block is considered). Then, the abstract collecting semantics collects initial type of a program. An example is given below. 


$$
\begin{aligned}
& \text { e ::= type } x \\
& \text { (type) } x \\
& S \llbracket \text { type } x \rrbracket=\lambda m .(m[x]=\langle S \llbracket \text { type } \rrbracket m, \perp\rangle) \\
& S \llbracket(\text { type }) x \rrbracket=\lambda m . i f(\text { type }(x) \sqsubseteq \text { type }) \\
& \text { then }(m[x]=\langle\text { type, } m(x)\rangle) \\
& x=e x p \\
& \text { if be else e } \\
& \text { while be } \\
& e ; e \\
& \{e\} \\
& \text { type } f(x) e \\
& \text { return exp } \\
& S \llbracket x=e x p \rrbracket=\lambda m .(m[x]=\langle t y p e(x), S \llbracket e x p \rrbracket m\rangle) \\
& S \llbracket \text { if } b e_{1} \text { else } e_{2} \rrbracket=\lambda m \text {.if } S \llbracket b \rrbracket \text { then } S \llbracket e_{1} \rrbracket m \text { else } S \llbracket e_{2} \rrbracket m \\
& S \llbracket \text { while } b e \rrbracket=\lambda m \text {.lfp }(\lambda X . \text { if }(S \llbracket b \rrbracket m) \text { then } S \llbracket e ; X \rrbracket m) \\
& S \llbracket e_{1} ; e_{2} \rrbracket=\lambda m . S \llbracket e_{2} \rrbracket\left(S \llbracket e_{1} \rrbracket m\right) \\
& S \llbracket\{e\} \rrbracket=\lambda m . S \llbracket e \rrbracket m \\
& S \llbracket \text { type }_{1} f\left(\text { type }_{2} x\right) e \rrbracket=\lambda m .\left(m[f]=\left\langle\text { type }_{1},\right.\right. \\
& \left.\left.\lambda x . S \llbracket \text { type }_{2} x^{\prime} ; x^{\prime}=x ; e \rrbracket m\right\rangle\right) \\
& \exp ::=x \\
& \text { | } \exp +\exp \\
& S \llbracket \text { returnexp } \rrbracket=\lambda m . S \llbracket e x p \rrbracket m \\
& S \llbracket x \rrbracket=\lambda m \cdot m(x) \\
& \text { value } \\
& S \llbracket \exp _{1}+\exp _{2} \rrbracket=\lambda m \cdot\left(S \llbracket \exp _{1} \rrbracket m+S \llbracket \exp _{2} \rrbracket m\right) \\
& S \llbracket \text { value } \rrbracket=\lambda m . v a l(\text { value })
\end{aligned}
$$

Fig. 7. Simple Imperative Language and its Semantics

$$
\begin{aligned}
& e::=\text { type } x \\
& \text { | (type) } x \\
& T \llbracket \text { type } x \rrbracket=\lambda h .(h[x]=T \llbracket \text { type } \rrbracket h) \\
& \text { | } x=\exp \\
& \text { if be elsee } \\
& \text { while be } \\
& \text { I } e ; e \\
& \{e\} \\
& T \llbracket(\text { type }) x \rrbracket=\lambda h . i f(h(x) \sqsubseteq \text { type }) \\
& \text { then }(h[x]=\text { type }) \\
& T \llbracket x=\exp \rrbracket=\lambda h .(h[x]=T \llbracket \exp \rrbracket h) \\
& \text { type } f(x) e \\
& T \llbracket \text { if b }_{1} \text { else } e_{2} \rrbracket=\lambda h .\left(T \llbracket e_{1} \rrbracket h \cup T \llbracket e_{2} \rrbracket h\right) \\
& T \llbracket \text { while } b e \rrbracket=\lambda h . T \llbracket e \rrbracket h \\
& T \llbracket e_{1} ; e_{2} \rrbracket=\lambda h . T \llbracket e_{2} \rrbracket\left(T \llbracket e_{1} \rrbracket h\right) \\
& T \llbracket\{e\} \rrbracket=\lambda h . T \llbracket e \rrbracket h \\
& \text { returnexp } \\
& T \llbracket \text { type }_{1} f\left(\text { type }_{2} x\right) e \rrbracket=\lambda h .\left(h[f]=\text { type }_{1}\right. \\
& \left.\cup T \llbracket \text { type }_{2} x^{\prime} ; x=x^{\prime} ; e \rrbracket h\right) \\
& \exp ::=x \\
& \text { | } \exp +\exp \\
& T \llbracket \text { return exp } \rrbracket=\lambda h . T \llbracket \exp \rrbracket h \\
& T \llbracket x \rrbracket=\lambda h \cdot h(x) \\
& \text { | value } \\
& \begin{aligned}
T \llbracket \exp _{1}+\exp _{2} \rrbracket & =\lambda h .\left(T \llbracket \exp _{1} \rrbracket h \sqcup T \llbracket \exp _{2} \rrbracket h\right) \\
T \llbracket \text { value } \rrbracket & =\text { typing }(\text { value })
\end{aligned}
\end{aligned}
$$

Fig. 8. Its Abstract Semantics of Types

Figure 9 presents such a process resulting in a hierarchical clustering of variables. Example:

$$
\begin{aligned}
& -\operatorname{dectype}(\mathrm{file} . \mathrm{c} / \mathrm{main} / \mathrm{t})=\text { char } \\
& -\operatorname{dec} \operatorname{var}(\mathbf{c h a r})=\{\mathrm{file} . \mathrm{c} / \mathrm{x}, \ldots, \mathrm{file} . \mathrm{c} / \mathrm{main} / \mathrm{t}, \ldots\}
\end{aligned}
$$

Type clustering is an interesting analysis but quite a basic one. It should be taken as starting points for further investigations in the abstraction-space. Considering more complex data-types for example, one could manage to clusterise the source code in objects rather than in modules, reasoning on data-type dependences to gather together structures and procedures. Dynamic clustering may even be considered (dynamic partitioning [2], communication in mobile systems [15]). More information about types and Abstract Interpretation may be found in [5]. 


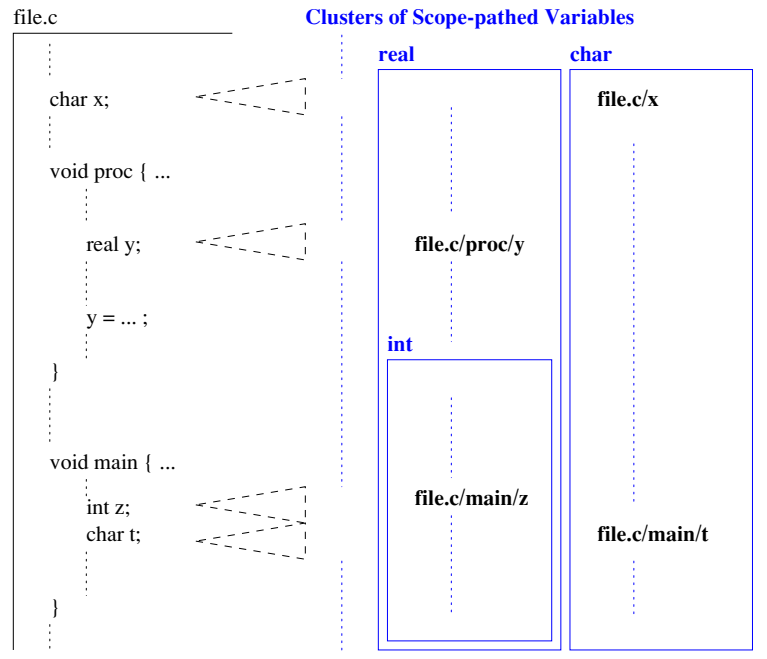

Fig. 9. Hierarchical Type Clustering.

\section{Related Work}

Automatic clustering is widely researched in system reconstruction. A survey can be found in [17]. A comparison with techniques like [12,11] gives some insights: in [11], an automatic modularisation technique is presented based on measures of information in cluster of code modules (intra-connectivity) and between clusters (inter-connectivity). The deal is to maximize the first measure while minimizing the later one. The results of these measures are combined in a function (Modularisation Quality or MQ) and the aim is to optimize it; the (sub)optimal solution being the clustering of modules we are looking for. In Abstract Clustering, intra-connectivity is replaced by subject domain (partial) order while inter-connectivity is described by abstract domain (partial) order. The modularisation quality function, $\mathrm{MQ}$, which combines intra and inter connectivity is substituted by a soundness requirement represented by an interpretation (a pair of adjoined functions - a Galois connection - between complete lattices). Thus, we keep the same architecture but lifted to a semantic level. Moreover, as shown in section 4, Abstract Clustering may be used as the basis of an analysis framework so called Abstract Clustering Framework.

\section{Conclusions}

Principles of Abstract Clustering have been introduced in section 2, developed in section 3 to define a generic clustering analysis, and structured in section 4 using an Abstract Clustering to build an Abstract Clustering Framework. Type clustering have been detailed as examples in section 5 and then section 6 compared our methodology to techniques of automatic modularisation of code. 
The applicability of the Abstract Clustering does not refers to a particular domain; the process that permits to define abstractions and then to refine analysis allows the user to draw its own path, from solution domains implemented in the source code to the problem domain. This method is general (analysis are built no embedded) and flexible (it can be easily adapted to be used in a variety of situations). Solutions may be adapted by the user to exploit simplifying aspects of the problem. Abstract Clustering provides a formal environment with which one constructs more accurate abstractions for different semantic (and so problem) domains. The subject problem domain guides the user in the selection of the appropriate abstract clustering. The Abstract Clustering Framework is extensible in the sense that abstractions may be created, extended, composed. From this point of view, it is an open system. The end-user is able to design and to develop analysis techniques for specific tasks.

It is applicable to large systems and scalable through the meta-level organization of Abstract Clustering. This organization of abstractions can be seen as semantic search strategies that work on systems both horizontally - the current semantic domain of analysis - and vertically - the hierarchy of semantic domain present at the meta-level. Semantic-patterns can be extracted, partial systems can be incrementally investigated using forget abstractions that are identity

functions define on a sub-domain of the subject domain, and irrelevant parts can be ignored by semantic-forget to obtain more useful knowledge.

\section{References}

1. A. Arnould, P. Baudin, J.M. Collart, A. Pacalet, J.Raguideau, and D.Schoen. Assistance in software validation. In DAta System In Aerospace (DASIA'98), 1998.

2. F. Bourdoncle. Abstract interpretation by dynamic partitioning. Journal of Functional Programming, 2(4):407-435, 1992.

3. Patrick Cousot. Abstract interpretation based static analysis parameterised by semantics. In P. van Hentenryck, editor, Proceedings of the 4 th International Symposium on Static Analysis, SAS'97, volume 1302 of Lecture Notes in Computer Science, pages 388-394, Paris, 1997. Springer-Verlag.

4. Patrick Cousot. Constructive design of a hierarchy of semantics of a transition system by abstract interpretation. Electronic Notes in Theoretical Computer Science(6):25, 1997.

5. Patrick Cousot. Types as abstract interpretations. In Conference Record of POPL '97: The 24th ACM SIGPLAN-SIGACT Symposium on Principles of Programming Languages, pages 316-331, Paris, France, 15-17 January 1997.

6. Patrick Cousot and Radhia Cousot. Abstract interpretation: A unified lattice model for static analysis of programs by construction or approximation of fixpoints. In Conference Record of the Fourth ACM Symposium on Principles of Programming Languages, pages 238-252, Los Angeles, California, January 17-19, 1977. ACM SIGACT-SIGPLAN.

7. Patrick Cousot and Radhia Cousot. Systematic design of program analysis frameworks. In Conference Record of the Sixth Annual ACM Symposium on Principles of Programming Languages, pages 269-282. ACM, ACM, January 1979. 
8. Patrick Cousot and Radhia Cousot. Abstract interpretation frameworks. Journal of Logic and Computation, 2(4):511-547, August 1992.

9. Patrick Cousot and Radhia Cousot. Comparing the Galois connection and widening/narrowing approaches to abstract interpretation. In M. Bruynooghe and M. Wirsing, editors, Proceedings of the 4 th International Symposium on Programming Language Implementation and Logic Programming (PLILP '92), volume 631 of Lecture Notes in Computer Science, pages 269-295, Berlin, 1992. SpringerVerlag.

10. Christopher Fraser and David R. Hanson. A Retargetable C Compiler. AddisonWesley, 1995.

11. S. Mancoridis, B. S. Mitchell, C. Rorres, Y. Chen, and E. R. Gansner. Using automatic clustering to produce high-level system organizations of source code. In International Workshop on Program Comprehension (IWPC'98), pages 45-52, 1998.

12. Hausi A. Müller, Mehmet A. Orgun, Scott R. Tilley, and James S. Uhl. A Reverse Engineering Approach To Subsystem Structure Identification. John Wiley \& Sons, 1993.

13. Scott R. Tilley. A reverse-engineering environment framework. Technical Report CMU/SEI-98-TR-005, Carnegie Mellon University, Software Engineering Institute, April 1998.

14. A. Venet. Abstract cofibered domains: Application to the alias analysis of untyped programs. Lecture Notes in Computer Science, 1145:366-??, 1996.

15. Arnaud Venet. Automatic determination of communication topologies in mobile systems. In Springer-Verlag, editor, Fifth International Symposium on Static Analysis (SAS'98), Berlin, Germany, 1998.

16. Steven J. Vickers. Topology Via Logic, volume 5 of Cambridge Tracts in Theoretical Computer Science. Cambridge University Press, 1988.

17. T. Wiggerts. Using clustering algorithms in legacy system remodularization. In Working Conference on Reverse Engineering, 1997. 